\title{
Prefrontal Corticotropin-Releasing Factor (CRF) Neurons Act Locally to Modulate Frontostriatal Cognition and Circuit Function
}

\author{
(DSofiya Hupalo, Andrea J. Martin, Rebecca K. Green, David M. Devilbiss, and Craig W. Berridge \\ Department of Psychology, University of Wisconsin-Madison, Madison, Wisconsin 53706
}

The PFC and extended frontostriatal circuitry support higher cognitive processes that guide goal-directed behavior. PFC-dependent cognitive dysfunction is a core feature of multiple psychiatric disorders. Unfortunately, a major limiting factor in the development of treatments for PFC cognitive dysfunction is our limited understanding of the neural mechanisms underlying PFC-dependent cognition. We recently demonstrated that activation of corticotropin-releasing factor (CRF) receptors in the caudal dorsomedial PFC (dmPFC) impairs higher cognitive function, as measured in a working memory task. Currently, there remains much unknown about CRFdependent regulation of cognition, including the source of CRF for cognition-modulating receptors and the output pathways modulated by these receptors. To address these issues, the current studies used a viral vector-based approach to chemogenetically activate or inhibit PFC CRF neurons in working memory-tested male rats. Chemogenetic activation of caudal, but not rostral, dmPFC CRF neurons potently impaired working memory, whereas inhibition of these neurons improved working memory. Importantly, the cognition-impairing actions of PFC CRF neurons were dependent on local CRF receptors coupled to protein kinase A. Additional electrophysiological recordings demonstrated that chemogenetic activation of caudal dmPFC CRF neurons elicits a robust degradation of task-related coding properties of dmPFC pyramidal neurons and, to a lesser extent, medium spiny neurons in the dorsomedial striatum. Collectively, these results demonstrate that local CRF release within the caudal dmPFC impairs frontostriatal cognitive and circuit function and suggest that CRF may represent a potential target for treating frontostriatal cognitive dysfunction.

Key words: corticotropin-releasing factor; DREADDs; in vivo electrophysiology; prefrontal cortex; striatum; working memory

Significance Statement

The dorsomedial PFC and its striatal targets play a critical role in higher cognitive function. PFC-dependent cognitive dysfunction is associated with many psychiatric disorders. Although it has long-been known that corticotropin-releasing factor (CRF) neurons are prominent within the PFC, their role in cognition has remained unclear. Using a novel chemogenetic viral vector system, the present studies demonstrate that PFC CRF neurons impair working memory via activation of local PKA-coupled CRF receptors, an action associated with robust degradation in task-related frontostriatal neuronal coding. Conversely, suppression of constitutive PFC CRF activity improved working memory. Collectively, these studies provide novel insight into the neurobiology of cognition and suggest that CRF may represent a novel target for the treatment of cognitive dysfunction.

\section{Introduction}

The prefrontal cortex (PFC) supports a diversity of "executive" cognitive processes that guide goal-directed behavior (Fuster,

Received Oct. 18, 2018; revised Dec. 11, 2018; accepted Jan. 4, 2019.

Author contributions: S.H. wrote the first draft of the paper; C.W.B. edited the paper. S.H. and C.W.B. designed research; S.H., A.J.M., and R.K.G. performed research; D.M.D. contributed unpublished reagents/analytic tools; S.H. analyzed data.

This work was supported by National Institutes of Health Grants MH102211, MH116526, and MH107140 and the Office of the Vice Chancellor for Research and Graduate Education at the University of Wisconsin-Madison with funding from the Wisconsin Alumni Research Foundation.

D.M.D. has ownership interest in NexStep Biomarkers, LLC. and Cerora, Inc. The remaining authors declare no competing financial interests.
2015). Many psychiatric disorders are associated with dysregulation of PFC-dependent cognition (Cubillo et al., 2010; Liston et al., 2011). Most drugs used to treat these disorders target catecholamine neurotransmitters. However, there are limitations to these drugs, including a lack of efficacy across the broader population and, in the case of attention deficit hyperactivity disorder

Correspondence should be addressed to Craig W. Berridge at berridge@wisc.edu.

S. Hupalo's present address: National Institute of Neurological Disorders and Stroke, Bethesda, MD 20892.

D.M. Devilbiss' present address: Department of Cell Biology \& Neuroscience, Rowan University School of Osteopathic Medicine, Stratford, NJ 08084

https://doi.org/10.1523/JNEUROSCI.2701-18.2019

Copyright $\odot 2019$ the authors $\quad 0270-6474 / 19 / 392080-11 \$ 15.00 / 0$ 
(ADHD), the fact that first-line treatments (psychostimulants) possess risk for abuse (Compton and Volkow, 2006; Setlik et al., 2009; Berridge and Arnsten, 2015). Thus, there is a need for improved treatments for PFC-dependent cognitive dysfunction. Unfortunately, the development of alternative treatments is impeded by a scarcity of noncatecholamine targets.

One potential target is corticotropin-releasing factor (CRF). It has long-been known that CRF neurons and receptors are prominent in the PFC (Swanson et al., 1983; De Souza et al., 1985). However, despite decades of research on the neurobiology of subcortical CRF systems, the functional significance of PFC CRF has been overlooked. We recently demonstrated that CRF receptor activation in the caudal dorsomedial PFC (dmPFC) of rats disrupts, whereas blockade of these receptors improves, spatial working memory (Hupalo and Berridge, 2016). These observations indicate that CRF acts in the PFC to regulate higher cognitive function. However, little is currently known about the neurobiology underlying the cognitive actions of PFC CRF neurotransmission, including fundamental issues, such as the source of CRF to the PFC and the output pathways impacted by PFC CRF signaling.

One possible source of CRF for cognition-modulating receptors are local CRF-synthesizing neurons (Olschowka et al., 1982; Swanson et al., 1983; Merchenthaler, 1984; Charlton et al., 1987; Lewis et al., 1989). The current study examined the cognitive actions of PFC CRF neurons using a novel dual-virus chemogenetic approach to bidirectionally manipulate these neurons in WT rats tested in a working memory task. For this, Credependent "excitatory" (hM3Dq) or "inhibitory" (hM4Di) designer receptors exclusively activated by designer drugs (DREADDs) are combined with CRF promoter-specific expression of Cre recombinase. We observed that chemogenetic activation of caudal, but not rostral, dmPFC CRF neurons impaired, whereas inhibition of caudal dmPFC CRF neurons improved, working memory. Additional studies demonstrated that the cognition-impairing effects of caudal dmPFC CRF neurons were dependent on local CRF receptors coupled to protein kinase A (PKA) signaling.

The dmPFC projects to the dorsomedial striatum (dmSTR), forming frontostriatal circuits that support higher cognition (Voorn et al., 2004; Mailly et al., 2013). Importantly, both nodes of this circuit are necessary for successful working memory (Spencer et al., 2012). Within the PFC, subpopulations of pyramidal neurons encode a diversity of information required for successful goal attainment, as measured in tests of working memory (Curtis and D'Esposito, 2003; Histed et al., 2009; Rigotti et al., 2013). Limited observations suggest that similar neural coding mechanisms exist within cognition-related striatal regions (Schultz and Romo, 1988; Levy et al., 1997; Akhlaghpour et al., 2016).

The working memory modulatory actions of PFC catecholamines are associated with a robust modulation of taskrelated coding properties of PFC neurons (Arnsten, 2011). The extent to which these neurophysiological actions are relayed to the downstream striatum is currently unclear. To determine whether PFC CRF neurotransmission impacts task-related activity in either the dmPFC or dmSTR, we combined ensemble single-unit recordings with chemogenetic activation of caudal dmPFC CRF neurons in working memory-tested animals. Activation of caudal dmPFC CRF neurons robustly degraded taskrelated coding properties of putative pyramidal $\mathrm{dmPFC}$ neurons, with lesser, though significant, effects observed for putative medium spiny neurons in the dmSTR.
Together, these studies provide the first evidence that PFC CRF neurons potently disrupt frontostriatal cognitive and circuit function. Additionally, these observations provide a strong rationale for future research into the behavioral and physiological actions of PFC CRF neurons, the neural mechanisms subserving these actions, and the potential clinical relevance of the PFC CRF system.

\section{Materials and Methods}

\section{Animals}

Male Sprague Dawley rats (300-500 g; Charles River) were pair-housed with enrichment (Nylabone, Bio-Serv) on a 13/11 h light/dark cycle. Animals used for electrophysiological recordings were singly housed. Animals were fed ad libitum for 4-7 d, and food was titrated for each animal (15-17 g chow/d) to maintain motivation for food reward while avoiding weight loss. Training/testing was conducted between

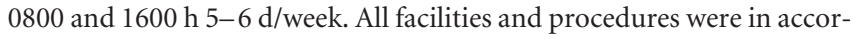
dance with National Institutes of Health guidelines and approved by an Institutional Animal Care and Use Committee.

\section{Working memory training}

Training and testing were conducted as previously described (Berridge et al., 2012b; Hupalo and Berridge, 2016). Briefly, animals were trained to enter the arm of a T-maze not chosen on the previous trial to receive food reward (45 mg sucrose pellet/trial, Bio-Serv). Between trials, animals were placed in a start box at the base of the maze and prevented from exiting by a gate (see Fig. $2 A$ ). Following surgery, animals resumed testing with the introduction of a delay between trials titrated to achieve the desired performance level. Sessions consisted of 20 trials (one session/d). For electrophysiology studies, sessions consisted of 30 trials (two sessions/d).

\section{Surgery}

Viral infusions. Rats were anesthetized with isoflurane and received a mixture of two viruses (see Fig. $1 A$ ) targeting the rostral (anteroposterior 4.0 ; lateral \pm 0.8 ; ventral -2.0 from brain surface) or caudal (anteroposterior 2.6; lateral \pm 0.8 ; ventral -3.0$)$ dmPFC: AAV8-CRF-Cre $(6.5 \times$ $10^{13} \mathrm{gc} / \mathrm{ml}$; Vector Biolabs) + AAV8-hSyn-DIO-hM3Dq-mCherry $\left(4.6 \times 10^{12} \mathrm{gc} / \mathrm{ml}\right.$; Addgene) or AAV8-hSyn-DIO-hM4Di-mCherry $\left(4 \times 10^{12} \mathrm{gc} / \mathrm{ml}\right)$ or AAV8-hSyn-DIO-mCherry $\left(3.1 \times 10^{12} \mathrm{gc} / \mathrm{ml}\right)$. The viral mixture was premixed at a 1:2 ratio of CRF-Cre to DIO-mCherry and infused into each dmPFC hemisphere at a rate of $0.25 \mu \mathrm{l} / \mathrm{min}$. Injector needles were left in place for $3 \mathrm{~min}$ before removal.

Cannulae. A subset of caudal dmPFC CRF-hM3Dq animals was implanted with cannulae (26 gauge) bilaterally over this region (anteroposterior 3.0-2.4; lateral \pm 0.8 ; ventral -0.2 ). Another group of caudal dmPFC CRF-hM3Dq animals were bilaterally cannulated targeting the medial septum (MS; anteroposterior 0.5 ; lateral \pm 0.9 with $4^{\circ}$ angle; ventral -0.5$)$.

Electrodes. Additional caudal dmPFC CRF-hM3Dq or control virus animals were implanted with chronic recording electrodes targeting both the dmPFC and dmSTR (see Fig. 4A), as previously described (Devilbiss et al., 2012, 2017). In the PFC, a linear electrode array (8 electrodes/array, $50 \mu \mathrm{m}$ wires, $\sim 200 \mu \mathrm{m}$ separation; NB Labs) was implanted into layer $\mathrm{V}$, oriented rostrocaudally (anteroposterior 3.6-2.2, lateral \pm 0.75 ; ventral -3.0). An 8-electrode bundle was unilaterally implanted into the dmSTR (anteroposterior 0.45 ; lateral \pm 3.5 with $11.5^{\circ}$ angle; ventral -3.6 ; see Fig. $5 A$ ).

Cannulae and electrodes were secured in place with skull screws and dental acrylic.

\section{Drugs}

CRF (human/rat, Bachem) and the PKA inhibitor, Rp-cAMPs (Tocris Bioscience), were dissolved in buffered artificial extracellular fluid (147 mм NaCl, 1.3 mм CaCl, 0.9 mм MgCl, 2.5 mм KCl, pH 7.4). D-Phe-CRF (human/rat, Bachem) and clozapine N-oxide (CNO; NIMH Chemical Synthesis and Drug Supply Program) were dissolved in 0.9\% saline. Before treatment, $\mathrm{CNO}$ was gently warmed to ensure the drug was in solution. 


\section{Experimental design}

Drug treatments. For systemic $\mathrm{CNO}$ treatments, animals were injected subcutaneously $(1 \mathrm{ml} / \mathrm{kg}) 45 \mathrm{~min}$ before testing. Bilateral intra-PFC ( $3 \mathrm{~mm}$ projection) and intra-MS (5.5 $\mathrm{mm}$ projection) $\mathrm{CNO}$ infusions were performed with 33-gauge needles using a microprocessorcontrolled pump (Harvard Apparatus) at a rate of 0.25 and $0.125 \mu \mathrm{l} / \mathrm{min}$ for $2 \mathrm{~min}$, respectively. Needles were kept in place for $2 \mathrm{~min}$ following infusions. Smaller infusion volumes $(250 \mathrm{nl}$ vs $500 \mathrm{nl}$ ) were used for the MS to minimize drug flow up the needle tract and into the lateral ventricles. Prior studies demonstrate this volume allows selective targeting of this region with multiple pharmacological manipulations (Berridge et al., 2012a).

For dual intra-PFC and systemic treatments, animals first received intra-PFC infusion of D-Phe-CRF, Rp-cAMPs, or vehicle followed by a subcutaneous $\mathrm{CNO}$ or vehicle injection $(1 \mathrm{ml} / \mathrm{kg})$. Animals were tested 30 min later.

Working memory testing procedures. Baseline performance levels of $80 \%-92.5 \%$ or $70 \%-82.5 \%$ were used to detect working memory impairing and improving effects, respectively. Baseline performance was calculated by averaging an animal's performance accuracy on the $2 \mathrm{~d}$ immediately before the treatment day. Given performance improves with repeated testing, delays were increased (10-30 s) when performance exceeded the desired range. In this study, no animals exceeded performance criteria past $30 \mathrm{~s}$ delays. Animals receiving intratissue infusions received a mock injection and/or intratissue infusion before the first treatment.

Histology and immunohistochemistry. Animals were anesthetized and transcardially perfused with $250 \mathrm{ml}$ chilled heparinized saline (1 unit heparin $/ \mathrm{ml} 0.9 \%$ saline) followed by $450 \mathrm{ml}$ of $4 \%$ PFA in $0.01 \mathrm{M} \mathrm{PB}, \mathrm{pH}$ 7.4. For electrophysiology studies, cathodal $15 \mu \mathrm{A}$ current was first passed through every electrode for $10 \mathrm{~s}$ before perfusion. Brains were removed, stored in PFA overnight, and taken through graded sucrose solutions (20\%-30\% sucrose in $0.1 \mathrm{~m}$ PBS, pH 7.4). Electrode or needle placement was verified in $40-\mu \mathrm{m}$-thick coronal sections stained with Neutral Red dye (Sigma-Aldrich).

For immunohistochemical processing of CRF and Fos, sections were rinsed with $0.01 \mathrm{M}$ PBS and incubated in a blocking agent containing $5 \%$ normal donkey serum in $0.01 \mathrm{M}$ PBS $+0.1 \%$ Triton X-100 at room temperature for $45 \mathrm{~min}$. Subsequently, the blocking agent was removed, and sections were incubated in primary guinea pig anti-CRF antibody (1:4000; catalog \#t-5007; Peninsula Labs) or rabbit anti-fos (1:3000; catalog \#abe457, EMD Millipore) in the blocking agent overnight at $4^{\circ} \mathrm{C}$. Sections were washed and incubated in secondary donkey anti-guinea pig AF488 or donkey anti-rabbit AF405 for $1.5 \mathrm{~h}$ at room temperature (Jackson ImmunoResearch Laboratories). Secondary-only controls were used in all instances. Sections were mounted and coverslipped using ProLong Diamond Antifade Mountant (Thermo Fisher Scientific). The primary antibodies used to target CRF and Fos have been extensively validated using preabsorption and other controls (Das et al., 2007; Rajbhandari et al., 2015; Scharner et al., 2016).

Viral spread and cell counts. The spread of viral expression within the PFC was determined using $40 \mu \mathrm{m}$ coronal sections. Immunohistochemical and cell counting procedures were used to assess (1) the proportion of viral-transfected CRF neurons and (2) the extent to which our viral vector system permitted activation of PFC CRF neurons using Fos immunoreactivity (-ir). Brain sections from animals receiving viral infusions were examined using an Olympus BX51 light and reflected fluorescence microscope. The spread of virus was mapped throughout the rostrocaudal and dorsoventral extent of the PFC to ensure that the majority of viral expression was restricted to one quadrant (rostral vs caudal dmPFC). For animals receiving intra-PFC viral infusions and intra-PFC/MS cannulae, alternating sections were Nissl stained to confirm injector needle placement while intervening sections were used to measure viral spread. Data were included only when histological analyses verified accurate placement of the reporter protein (mCherry) and minimal tissue damage.

In a subset of animals, single-labeled (CRF-ir/Fos-ir or mCherry) and double-labeled (CRF-ir/Fos-ir + mCherry) neurons were counted in $600 \times$ images using a Nikon A1R + confocal microscope. For each sec- tion, 3 or 4 images were obtained from the main body of viral expression of each hemisphere. Images were obtained from 3 to 5 sections/animal (4 total animals). Cells were only counted if there was a clear nucleus. The percentage of double-labeled mCherry neurons was averaged. Cells were considered double-labeled only when fluorescence was clearly observed for mCherry (red signal, cytoplasm) and CRF-ir (green signal, cytoplasm) or Fos-ir (UV/blue signal, nucleus) within the same cell.

Electrophysiological recordings in working memory tested rats. On testing days, animals were transported to the recording room and tethered to a 32-channel commutator and a multichannel electrophysiology acquisition processor (MAP, Plexon). Putative single units in the dmPFC and dmSTR were isolated using template-matching algorithms to discriminate action potentials. Extracellular action potential waveforms exhibiting at least a 3:1 signal-to-noise ratio were discriminated using template-matching algorithms. The following criteria ensured that sorted waveforms arose from single neurons: (1) variability of peak waveform voltage, (2) variability of waveform slope, (3) scattergram distribution in the first three principal components, and (4) refractory period (see Fig. 4C). For all recording sessions, neural activity was simultaneously amplified, discriminated, and time stamped as previously described (Devilbiss et al., 2012, 2017).

After spike-sorting, a baseline recording session (30 trials, $10 \mathrm{~s}$ delays) was conducted. One hour later, animals were injected with CNO (3 mg/ $\mathrm{kg}$ ) or vehicle and $45 \mathrm{~min}$ later tested for an additional 30 trial session. Video recordings ( 80 frames/s) and IR beam grids time-stamped the animal's location in the T-maze (see Fig. $4 B$ ). When animals fully entered a T-maze arm (see Fig. 4A, pink quadrant), either a high- or lowfrequency $0.5 \mathrm{~s}$ tone identified correct and incorrect choices, respectively. Finally, the moment the animal bit on the sucrose pellet (reward) was time-stamped from video.

Neuron identification and event tuning. For the dmPFC, wide-spiking (WS), putative glutamatergic, versus narrow-spiking (NS), putative inhibitory cell types were differentiated by quantifying the peak-to-peak latency of the extracellular action potential waveform (WS > $200 \mu \mathrm{s} ; \mathrm{NS}$, $100-200 \mu \mathrm{s}$ ), as previously described (Povysheva et al., 2006; Mitchell et al., 2007; Devilbiss et al., 2017). For the dmSTR, medium spiny neurons (MSNs) and fast-spiking (FS) interneurons were identified using the peak-to-valley duration (MSN $>250 \mu \mathrm{s}$; FS $<200 \mu \mathrm{s}$ ) and firing rate (MSN < 8 Hz) (Berke et al., 2004; Kim et al., 2007; Stalnaker et al., 2012).

Distinct subpopulations of $\mathrm{dmPFC}$ and dmSTR neurons displayed excitatory responses to specific T-maze task events, including delay, reward, correct signal, and error signal (see Fig. $4 D, E$ ). Given the relatively high baseline task accuracy $(84.6 \pm 1.6 \%)$, it was not possible to analyze error responses of dmPFC or dmSTR neurons. The selective response or "event-tuning" of a neuron was determined during baseline recordings from the $z$ score of a neuron's activity during a particular task interval versus its overall spiking activity. $z$ scores $>0.025$ for the delay period and $>0.18$ for all other task events identified groups of neurons displaying strong tuning to task events, similar to exemplar responses described by other laboratories (Fuster and Alexander, 1971; Batuev et al., 1990; Horst and Laubach, 2013). Lower $z$ score thresholds were used for the delay interval given it was $\sim 10$-fold longer in duration than other task intervals. Units displaying $z$ scores between -0.18 and 0.08 ( -0.045 to 0.01 for the delay) were considered untuned to task events. Units with $z$ scores ranging between the cutoffs for strongly tuned and untuned neurons were excluded from analyses.

Consistent with prior studies in working memory tested rats (Hyman et al., 2010; Devilbiss et al., 2017), we observed no significant differences in baseline mean spiking rates for left versus right or correct versus incorrect trials among strongly tuned and untuned neurons in either region. Therefore, these task events/conditions were grouped together in subsequent analyses. To account for any differences in baseline spiking rates among neurons and experimental cohorts, and to accurately compare the effects of CNO on task-related responses, mean spike rates are presented as a percentage change from the baseline session.

\section{Statistical analyses}

Cognitive testing. A repeated-measures ANOVA analyzed the effect of drug treatments on performance accuracy. Statistical comparisons be- 
tween drug dose and vehicle were determined using Bonferroni corrected $t$ tests.

Electrophysiological recordings. The mean spiking rate during each task interval was determined on a trial-by-trial basis using perievent time histogram (PETH) analysis. For each neuron, the percentage change in mean spiking rate from baseline was determined for each task event and averaged for each neuron category (strongly tuned, untuned). No significant changes in firing rate from baseline were observed in vehicletreated hM3Dq animals or CNO-treated viral controls. Thus, changes in firing rate were analyzed using group (hM3Dq-CNO, hM3Dq-VEH, control-CNO) as a between-subject variable in a one-way ANOVA. Changes in population sizes of strongly tuned and untuned neurons were calculated using a $\chi^{2}$ test. Pairwise comparisons between the three groups were determined using Bonferroni corrected $t$ tests.

\section{Results}

\section{Chemogenetic targeting of CRF neurons}

To assess the working memory actions of PFC CRF neurons, "excitatory" (hM3Dq) or "inhibitory" (hM4Di) DREADDs were infused into the caudal or rostral dmPFC (Fig. 1A). An additional group received a control virus mixture lacking a DREADD transgene (CRF-Cre + DIO-mCherry). Robust reporter protein expression was observed within 3 weeks (Fig. 1B). Viral expression was limited to a radius of $\sim 500 \mu \mathrm{m}$, filling the majority of either the rostral or caudal dmPFC with minimal spread into the ventromedial PFC or adjacent dmPFC hemifields (Fig. 2Bi,Ci). The viral mixture transfected CRF neurons efficiently: $94 \pm 5 \%$ of mCherry neurons colocalized with CRF-ir $(n=354$ cells, 4 animals; Fig. 1C). Importantly, mCherry-positive cells lacking CRF-ir were not observed.

To assess whether the DREADD agonist, CNO, activates hM3Dq-expressing PFC CRF neurons, we examined Fos-ir in hM3Dq $(n=4)$ or viral control animals $(n=3)$ treated with 3 $\mathrm{mg} / \mathrm{kg} \mathrm{CNO}$ in the home-cage (highest dose used in subsequent studies). In hM3Dq animals, CNO elicited Fos-ir in $79 \pm 0.6 \%$ of mCherry neurons $(n=347$; Fig. $1 D i)$. In contrast, no Fos-ir was observed in mCherry neurons within the dmPFC of viral controls (Fig. 1Dii).

\section{Working memory actions of PFC CRF neurons}

Chemogenetic activation of caudal dmPFC CRF neurons (CRFCre + DIO-hM3Dq, $n=7$ ) dose-dependently impaired working memory performance (baseline, $83.9 \pm 2.1 \%$ ) relative to vehicle (baseline, $80.7 \pm 1.7 \% ; F_{(1,6)}=28.6, p=0.002$ ) and $\mathrm{CNO}$ treated viral controls (baseline, $81.7 \pm 2.8 \% ; n=7, F_{(1,13)}=35.0$, $p<0.001$; Fig. 2Bii). This occurred in the absence of significant changes in run time (vehicle: $3.6 \pm 1.0 \mathrm{~min} ; 3 \mathrm{mg} / \mathrm{kg} \mathrm{CNO}: 3.8 \pm$ $0.5 \mathrm{~min}, F_{(1,6)}=1.1, p=0.4$; viral controls: $3.9 \pm 1.0 \mathrm{~min}$, $\left.F_{(1,13)}=1.5, p=0.3\right)$. In contrast, chemogenetic activation of rostral dmPFC CRF neurons $(n=6)$ had no significant effects on performance (baseline, $86.7 \pm 1.7 \%$ ) relative to vehicle (baseline, $\left.84.2 \pm 2.1 \% ; F_{(1,5)}=0.9, p=0.39\right)$ or CNO-treated viral controls $\left(n=7 ; F_{(1,12)}=1.0, p=0.3\right.$; Fig. 2Cii).

In general, mCherry expression was not observed outside the targeted region of the PFC. The one exception to this was in $\sim 30 \%$ of animals receiving viral infusions in the caudal, but not rostral dmPFC, retrograde mCherry labeling of cell bodies was observed in the MS (Fig. 2Di), a region known to project to the PFC (Senut et al., 1989). To determine whether activation of MS CRF neurons could have contributed to the cognition-impairing effects of systemic CNO, a subset of animals containing mCherry in the MS (confirmed postmortem; $n=4$ ) were infused with $\mathrm{CNO}$ directly into the MS or caudal dmPFC. In these animals, CNO infusions $(0.5 \mathrm{~mm})$ into the caudal dmPFC impaired task performance comparable with that seen with systemic administration (baseline, $88.6 \pm 1.7 \% ; n=7, F_{(1,6)}=22.4, p=0.003$ ). In contrast, intra-MS CNO infusions had no significant effects on performance (baseline, $85.9 \pm 3.3 \% ; F_{(1,3)}=0.8, p=0.4$; Fig. 2Dii).

In contrast to that seen with activation of PFC CRF neurons, chemogenetic inhibition of caudal dmPFC CRF neurons (CRFCre + DIO-hM4Di) improved task performance (baseline, $78.1 \pm 1.1 \%$ ) relative to vehicle (baseline, $81.8 \pm 1.1 \% ; n=8$, $\left.F_{(1,7)}=15.6, p=0.006\right)$ or CNO-treated viral controls (baseline, $81.7 \pm 2.8 \% ; n=7, F_{(1,14)}=20.9, p<0.001$; Fig. $\left.2 E\right)$. This effect occurred in the absence of significant effects on maze run time (vehicle: $2.8 \pm 0.3 \mathrm{~min} ; 3 \mathrm{mg} / \mathrm{kg}$ CNO: $2.7 \pm 0.3 \mathrm{~min}, F_{(1,7)}=0.5$, $p=0.6$; viral controls: $\left.3.9 \pm 0.8 \mathrm{~min}, F_{(1,14)}=2.1, p=0.1\right)$.

\section{Circuit and intracellular signaling mechanisms underlying the cognitive actions of PFC CRF neurons}

Additional studies examined whether CRF neurons in the caudal dmPFC release CRF locally to impair working memory. Animals expressing CRF-hM3Dq in this region received infusions of either vehicle or the CRF antagonist, D-Phe-CRF (100 ng/hemisphere) directly into the caudal dmPFC before receiving systemic $\mathrm{CNO}$ at the maximally impairing dose ( $3 \mathrm{mg} / \mathrm{kg} ; n=9)$. In animals pretreated with intra-PFC vehicle, $\mathrm{CNO}$ elicited a robust working memory impairment (baseline, $86.9 \pm 1.8 \% ; F_{(1,8)}=$ $49.5, p<0.001)$ that was prevented by local D-Phe-CRF infusions (baseline, $82.6 \pm 1.9 \%$; $\mathrm{CNO} \times$ antagonist interaction, $F_{(1,8)}=$ 31.4, $p<0.001$; Fig. 3A).

CRF1 receptors are the dominant subtype in the rodent PFC and may couple to the Gs-PKA intracellular signaling cascade (Chalmers et al., 1995; Miguel et al., 2014). To examine whether the cognition-impairing actions of PFC CRF neurons are dependent on local PKA signaling, animals received bilateral infusions of vehicle or the PKA inhibitor, Rp-cAMPs (20 nM), into the caudal dmPFC before systemic CNO ( $3 \mathrm{mg} / \mathrm{kg} \mathrm{CNO} ; n=5$ ). Rp-cAMPs alone had no significant effects on task performance (Fig. $3 B$; baseline, $85.6 \pm 1.2 \% ; F_{(1,4)}=1.4, p=0.3$ ). In animals pretreated with intra-PFC vehicle, $\mathrm{CNO}$ impaired task performance (baseline, $88.5 \pm 1.3 \% ; F_{(1,4)}=29.4, p=0.005$ ), whereas intra-PFC Rp-cAMPs pretreatment completely blocked this effect (baseline, $85.6 \pm 2.1 \%$; CNO $\times$ Rp-cAMPs interaction; $F_{(1,4)}=27.2, p=0.01$; Fig. $\left.3 B\right)$.

\section{Effects of PFC CRF neuronal activation on task-related activity across the frontostriatal circuit}

To assess the effects of caudal dmPFC CRF neuronal activation on the coding properties of neurons within dorsomedial frontostriatal circuitry, animals were implanted with chronic recording electrodes in the dmPFC and dmSTR following intracaudal dmPFC infusion of CRF-hM3Dq $(n=5)$ or viral vector control animals $(n=3)$ (Fig. 4). Animals were tested for a 30 trial baseline session and a second 30 trial session following treatment with vehicle or CNO.

\section{Baseline neuronal response properties}

Across all animals, we isolated a total of $420 \mathrm{dmPFC}$ neurons (most within dorsal prelimbic PFC), 96\% classified as WS and 4\% as NS. Due to the low number of NS dmPFC neurons isolated, these neurons were not analyzed further. Under baseline conditions, $25 \%$ of dmPFC WS, putative pyramidal, neurons were classified as being strongly tuned to the delay interval, whereas $10 \%$ were strongly tuned to reward. Interestingly, dmPFC WS neurons were relatively insensitive to the correct signal tone and thus were not analyzed further. A larger proportion of dmPFC WS 
A

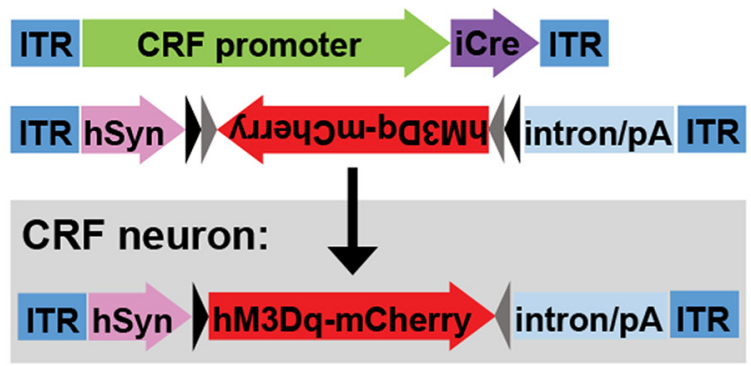

B

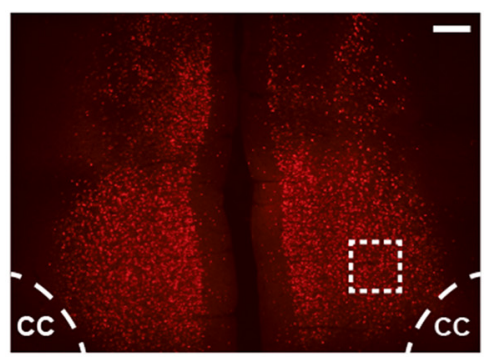

C
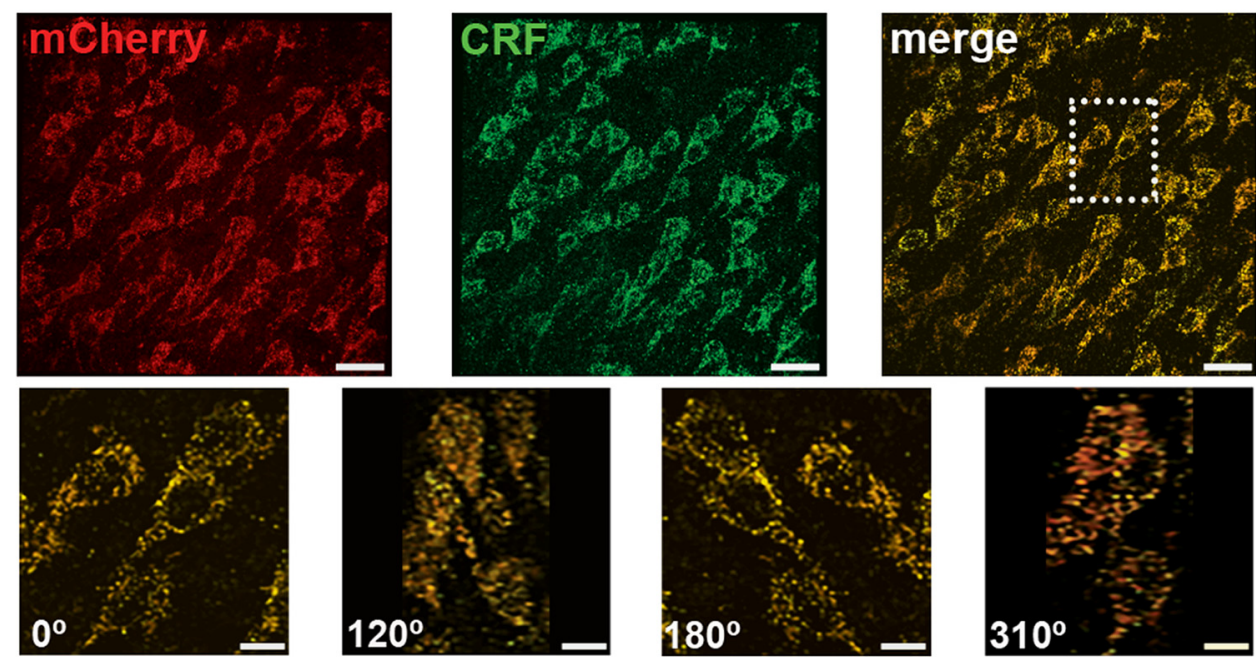

Di hM3Dq-mCherry + CNO

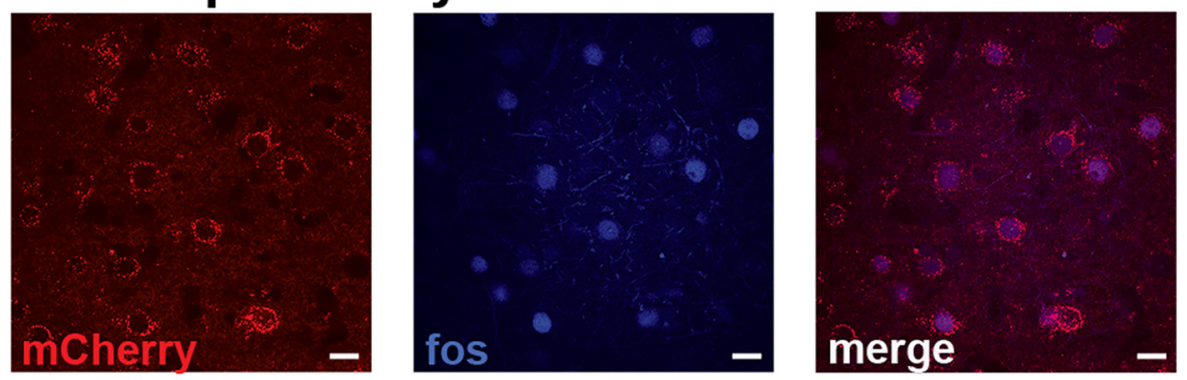

\section{Dii mCherry + CNO}

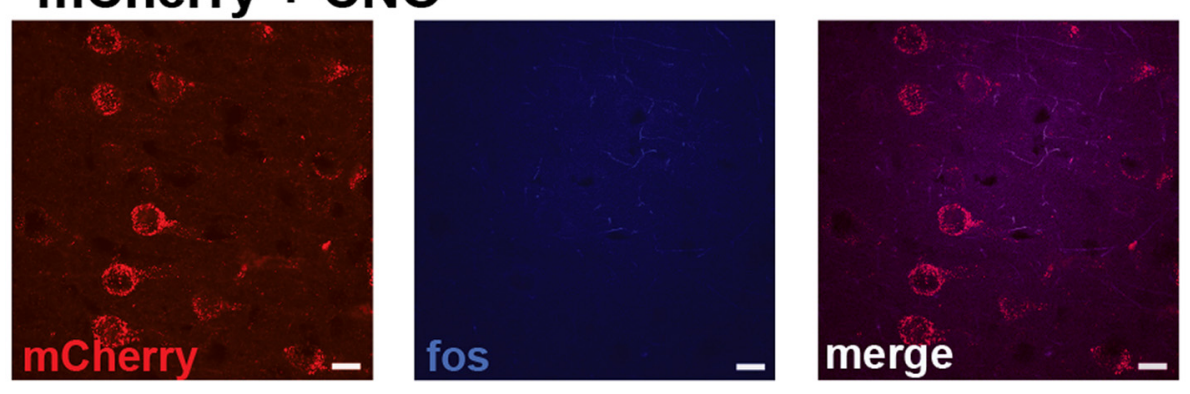

Figure 1. Chemogenetic approach. $\boldsymbol{A}$, Schematic depicting dual viral system to activate (CRF-Cre + hSyn-DIO-hM3Dq) or inhibit (CRF-Cre + hSyn-DI0-hM4Di) CRF neurons in the PFC. $\boldsymbol{B}$, Photomicrograph depicting mCherry expression in the caudal dmPFC from a CRF-hM3Dq-treated animal. Scale bar, $200 \mu \mathrm{m}$. cc, Corpus callosum. C, Top, Collapsed $30 \mu \mathrm{m} z$ stack from inset in $\boldsymbol{B}$, demonstrating mCherry colocalization with CRF-ir cells. Scale bar, $30 \mu \mathrm{m}$. Bottom, Inset from above, rotated at various angles. Scale bar, $10 \mu \mathrm{m}$. Di, CNO elicits an excitatory influence in PFC CRF neurons as measured by Fos-ir in mCherry neurons of CNO-treated CRF-hM3Dq animals. Dii, Absence of Fos-ir in the PFC of CNO-treated viral controls (mCherry + CNO; CRF-Cre hSyn-DI0-mCherry). Scale bar, 10 $\mu$ m.

neurons was classified as being insensitive ("untuned") to task events (delay, 39\%; reward, 53\%).

Within the dmSTR, we isolated a total of 255 neurons, $88 \%$ classified as MSN, 9\% as FS, and 3\% unclassified. Given the rel- atively small number of FS neurons, only MSNs were included in subsequent analyses. dmSTR MSN neurons also displayed strong delay-related (30\%) and reward-related (16\%) activity. In contrast to the dmPFC, a robust subpopulation of MSNs exhibited 
A

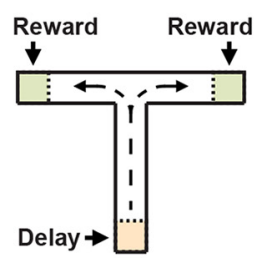

Di

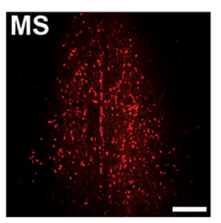

Bi

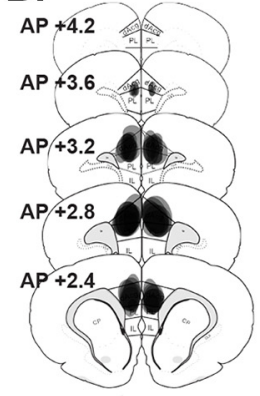

Bii caudal dmPFC

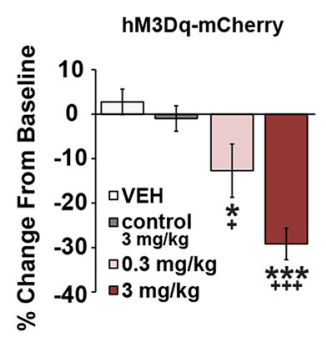

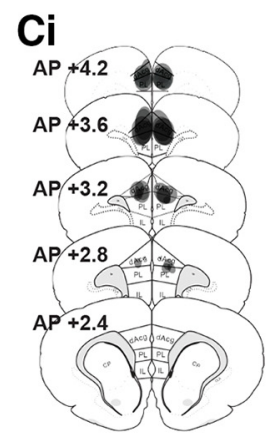

E

\section{Cii rostral dmPFC}

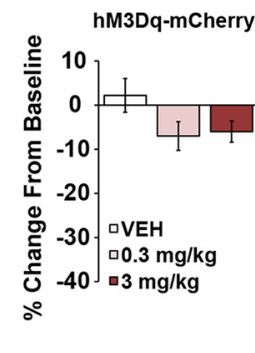

Dii
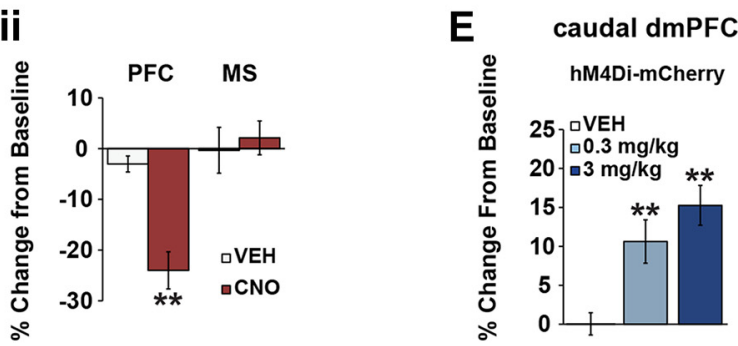

Figure 2. CRF neurons in the caudal, but not rostral, dmPFC modulate working memory. A, T-maze schematic. Bi, Schematics depict hM3Dq viral spread in the caudal dmPFC (anteroposterior 3.2-2.2) from all animals tested. Bii, CNO dose-dependently impairs task performance relative to vehicle ( $n=7)$ and CNO-treated viral control animals ( $n=7 ;$ control $3 \mathrm{mg} / \mathrm{kg})$. Ci, hM3Dq viral spread in the rostral dmPFC (anteroposterior 4.2-3.2). Cii, Chemogenetic activation of CRF neurons in the rostral dmPFC has no significant effects on task performance relative to vehicle ( $n=6$ ) and viral controls $(n=7)$. Di, Left, Retrograde mCherry cell body labeling observed in the MS in $\sim 30 \%$ of animals. Right, Schematics representing intra-MS infusion sites ( $n=4)$. Dii, When infused into the PFC, $0.5 \mathrm{~mm}$ CNO robustly impairs task performance $(n=7)$, while having no effects on performance when infused into the MS $(n=4)$. $E$, Chemogenetic suppression of CRF neurons in the caudal dmPFC improves task performance relative to vehicle $(n=8)$ and CNO-treated viral controls $(n=7)$. Results are mean \pm SEM percentage change in accuracy relative to baseline. ${ }^{*} p<0.05$ versus vehicle. ${ }^{+} p<0.001$ versus viral controls. ${ }^{* *} p<0.01$ versus vehicle. ${ }^{* * *} p<0.001$ versus vehicle. ${ }^{++} p<0.001$ versus viral controls.

A

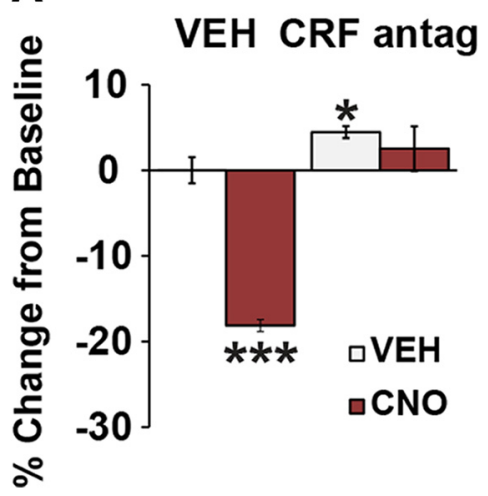

B

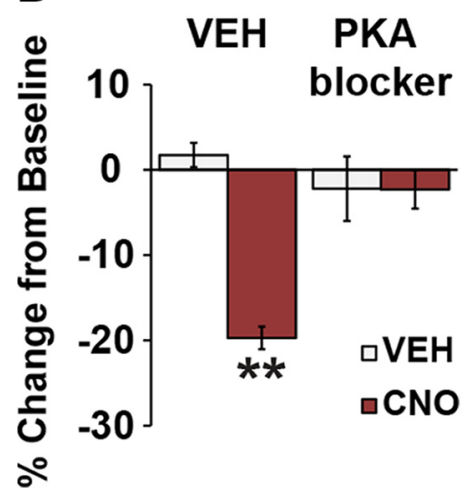

$24,54 \%$ reduction) relative to vehicle or CNO-treated viral controls (hM3DqVEH: $n=25,-0.6 \%$; control-CNO: $n=$ $54,0.1 \%$; Fig. $5 C$ ). Activation of PFC CRF neurons significantly diminished the population size of strongly tuned delay neurons by $75 \%$ relative to $\mathrm{hM} 3 \mathrm{Dq}-\mathrm{VEH}$ $(-8 \%)$ and control-CNO $(-30 \%)$ groups $\left(\chi^{2}=6.3, p=0.04\right.$; Fig. $\left.5 C\right)$. PFC CRF neuronal activation also significantly reduced reward-related activity of WS neurons $\left(F_{(2,39)}=3.1, p=0.05, n=16\right.$, $-29.1 \%)$ relative to vehicle $(n=15$, $-0.9 \%)$ and viral controls $(n=11,2.6 \%)$, while not significantly affecting the population size of strongly tuned reward neurons $\left(\chi^{2}=0.1, p=0.9\right.$; hM3Dq-CNO, $-19 \%$; hM3Dq-VEH, - 13\%; controlCNO, -27\%; Fig. 5C).

As shown in Figure 5D, PFC CRF neuronal activation had no significant effects prominent and punctate excitatory responses to the correct signal tone $(21 \%)$. A large proportion of MSNs were untuned to delay $(41 \%)$, reward $(72 \%)$, and correct signal (64\%).

Effects of caudal dmPFC CRF neuronal activation on task-related activity in the dmPFC

Vehicle treatment of hM3Dq animals $(n=4)$ or CNO treatment $(3 \mathrm{mg} / \mathrm{kg})$ of viral controls $(n=3)$ did not significantly affect working memory (hM3Dq-VEH: baseline, $84.3 \pm 1.9 \%$; $p=$ 0.14; control-CNO: baseline, $84.1 \pm 2.2 \%$; $p=0.16$; Fig. $5 B)$. In hM3Dq animals $(n=5)$, CNO significantly impaired task performance (baseline, $85.4 \pm 2.3 \% ; p=0.0001$; Fig. $5 B$ ). This was associated with a robust suppression of task-related activity of delay-tuned dmPFC WS neurons $\left(F_{(2,100)}=22.3, p<0.001, n=\right.$ on the larger population of WS neurons that were untuned to either delay (spiking: $F_{(2,157)}=1.1, p=0.3$; population size: $\chi^{2}=$ $0.7, p=0.7$ ) or reward (spiking: $F_{(2,213)}=0.23, p=0.8$; population size: $\chi^{2}=2.04, p=0.4$ ).

PFC CRF neuronal activation elicits a weaker degradation of task-related activity in the dmSTR

Chemogenetic activation of PFC CRF neurons robustly diminished the population size of delay-tuned MSNs $(-75 \%)$ relative to vehicle $(-7 \%)$ and $\mathrm{CNO}$-treated viral controls $\left(14 \% ; \chi^{2}=5.7\right.$, $p=0.05$; Fig. $6 A$ ). This was accompanied by a nonsignificant trend for reduction in delay-related activity $\left(F_{(2,65)}=1.3, p=0.2\right.$, $n=12,-18.4 \%)$ relative to vehicle $(-3.6 \%, n=28)$ and CNO- 
A

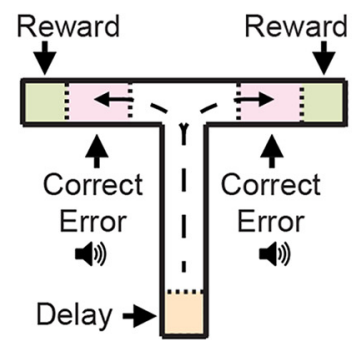

B

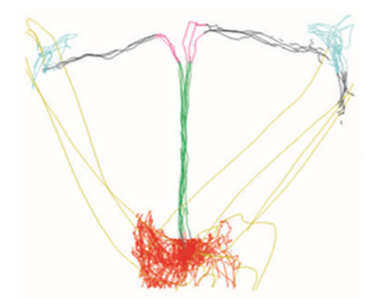

C

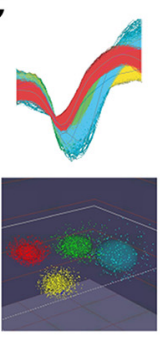

D dmPFC

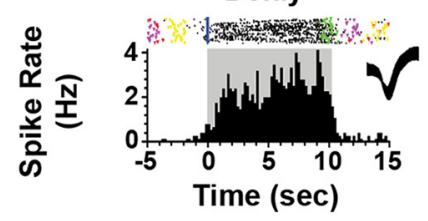

E dmSTR

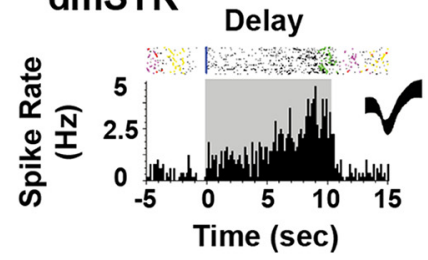

Correct Signal - (4))
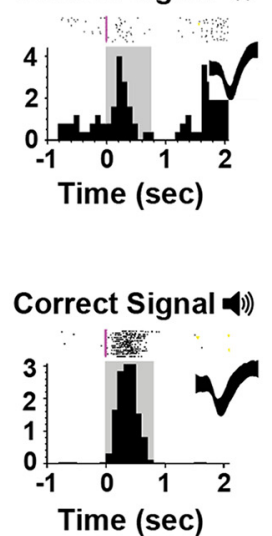

Error Signal (4))
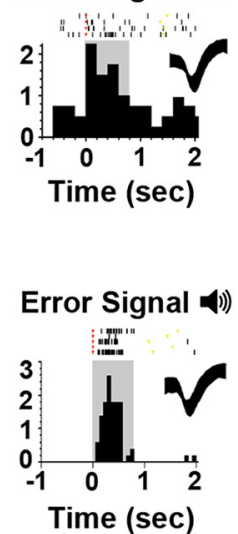

Reward

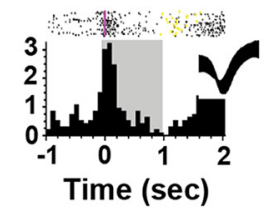

Figure 4. Frontostriatal recordings in working memory-tested rats. A, T-maze schematic illustrating task events, including delay, two distinct auditory tones serving as outcome-related signals on correct versus error trials, and sugar reward. $\boldsymbol{B}$, Animal position is tracked using video recordings and infrared beams. C, Top, Action potential waveforms of 4 discriminated WS $\mathrm{dm} P F($ neurons. Bottom, Waveforms from these units exhibit separable clusters in 3D-principal component space. D, Exemplar rasters (top) and PETHs (bottom) of WS dmPFC neurons exhibiting punctate excitatory responses during T-maze events. Shaded areas of PETHs represent duration of interval. Spiking rates were calculated for $100 \mathrm{~ms}$ time bins. E, Exemplar rasters and PETHs of putative MSNs within the dmSTR displaying strong task tuning.

treated viral controls ( $n=28,1.0 \%$; Fig. $6 A$ ). For reward-tuned MSNs, PFC CRF neuronal activation did not significantly affect task-related activity $\left(-21 \%, F_{(2,33)}=1.2, p=0.3, n=9 ; \mathrm{VEH}\right.$, $0 \%, n=20$; CNO-treated controls, $2.2 \%, n=19$ ), or population size $\left(\chi^{2}=0.01, p=0.9\right.$; Fig. $\left.6 A\right)$. MSNs strongly tuned to the correct signal were insensitive to $\mathrm{CNO}$ or vehicle across all groups (spiking: $F_{(2,45)}=0.03, p=0.9$; population size: $\chi^{2}=0.6, p=$ 0.7 ), as were MSNs untuned to delay (spiking: $F_{(2,89)}=0.5, p=$ 0.6 ; population size: $\chi^{2}=0.6, p=0.7$ ), reward (spiking: $F_{(2,158)}=0.06, p=0.9$; population size: $\left.\chi^{2}=0.9, p=0.6\right)$, and correct signal (spiking: $F_{(2,140)}=0.1, p=0.9$; population size: $\chi^{2}=1.1, p=0.5$; Fig. $\left.6 B\right)$.

\section{Discussion}

Both the PFC and CRF have long been implicated in psychopathology associated with cognitive impairment. Given this, it is surprising that the cognitive actions of CRF neurotransmission in the PFC have been largely overlooked. The current studies provide the first evidence that CRF neurons in the caudal dmPFC are constitutively active under working memory testing conditions and act to impair working memory via local PKA-coupled CRF receptors. Additionally, these studies demonstrate that the cognition-impairing action of CRF neurotransmission in the caudal dmPFC is associated with a robust degradation of delayand reward-related activity within the dmPFC and, to a lesser extent, in the dmSTR. Collectively, these observations provide novel insight into the neurobiology of frontostriatal-dependent cognition and suggest that CRF may represent a novel target for the treatment of PFC/frontostriatal-dependent cognitive dysfunction.
Topography of CRF actions within the dmPFC

The cognitive actions of PFC CRF neurons were topographically organized, being limited to the caudal portion of the dmPFC. This is consistent with a well-described functional topography of the rodent medial PFC dorsoventrally (Voorn et al., 2004; Gabbott et al., 2005) and rostrocaudally (Alsene et al., 2011; Hupalo and Berridge, 2016). The neural bases for the topography associated with the cognitive actions of PFC CRF are currently unknown. Given CRF neurons and receptors are present throughout the medial PFC (Swanson et al., 1983; De Souza et al., 1985), this topography does not appear to arise from differential neuronal and/or receptor distribution. Instead, this may reflect a topographical organization in the projection patterns of PFC neurons. Recent studies indicate that CRF-synthesizing and CRF receptor-expressing neurons in the rodent $\mathrm{PFC}$ represent distinct neuronal populations (Uribe-Mariño et al., 2016). Moreover, at least some of these CRF receptor-expressing neurons are pyramidal neurons (Gallopin et al., 2006). Thus, the restriction of the cognitive effects of CRF neurotransmission to the caudal dmPFC may reflect unique projection patterns of CRF receptorexpressing pyramidal neurons from this region. Consistent with this, extensive evidence demonstrates differing efferent projection targets across dorsal versus ventral PFC subfields (Sesack et al., 1989; Heidbreder and Groenewegen, 2003; Gabbott et al., 2005). The topographical organization of rostral versus caudal PFC projections has been less intensively studied. However, limited observations indicate the rostral medial PFC extends denser projections to the rostroventral and dmSTR, whereas the caudal medial PFC more strongly innervates the caudal ventral striatum 
A

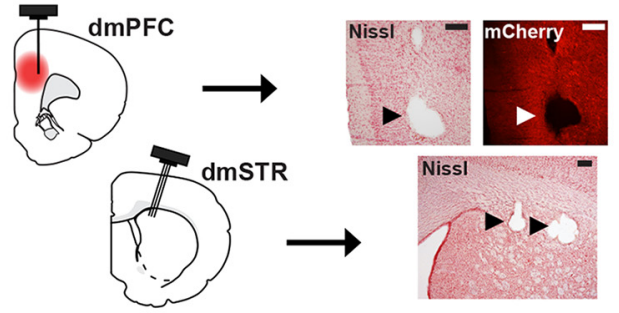

C Strongly tuned

Delay
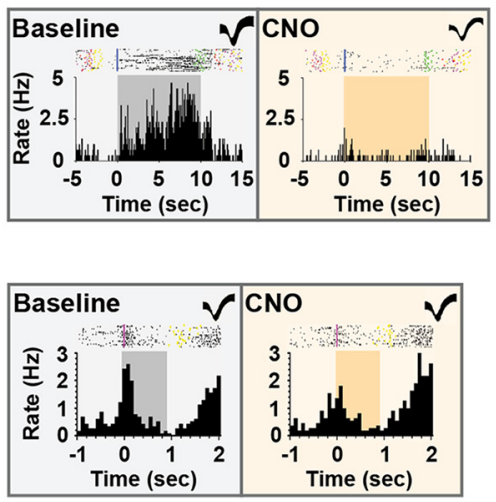

D Untuned

Delay
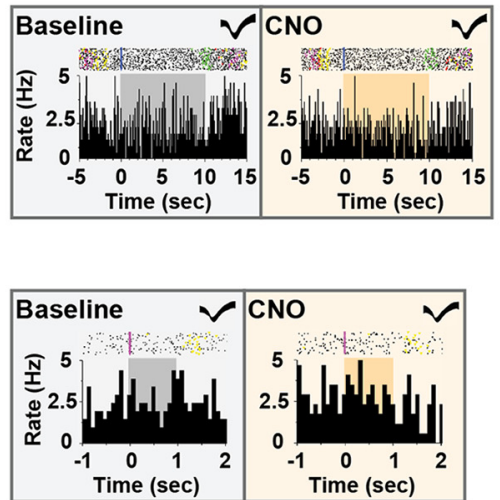

B Performance Accuracy

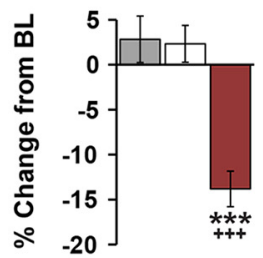

$\square$ control-CNO
$\square$ hM3Dq-VEH
$\square$ hM3Dq-CNO

Spiking Activity
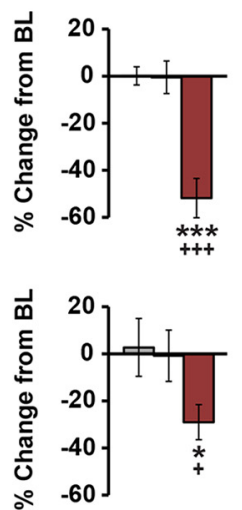

Spiking Activity
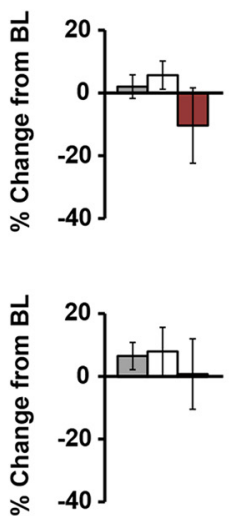

Population Size
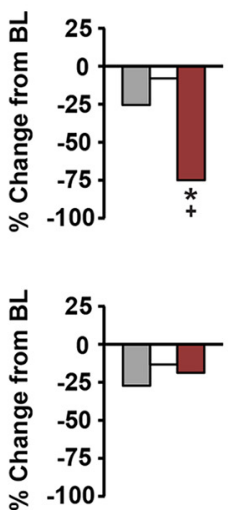

Population Size
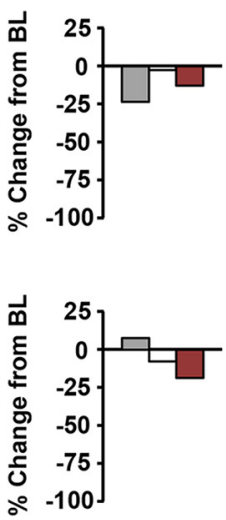

Figure 5. Chemogenetic activation of CRF neurons in the caudal dmPFC degrades delay and reward signaling in WS dmPFC neurons. A, Left, Schematics depicting CRF-hM3Dq expression in the caudal dmPFC and electrode placements in the dmPFC and dmSTR. Right, $4 \times$ photomicrographs depicting placement of one electrode in layer $V$ of the dmPFC and three electrodes in the dmSTR. Scale bar, $250 \mu \mathrm{m}$. B, In recorded animals, CNO impaired task performance in the hM3Dq group (5 animals, 7 recording sessions), but not in vehicle (4 animals, 10 sessions) or CN0-treated viral controls ( 3 animals, 10 recording sessions). C, Left, Exemplar rasters/PETHs demonstrating task-related activity of strongly tuned delay (top) and reward (bottom) WS neurons under baseline and CNO conditions (delay, $10 \mathrm{~s}$; reward, 1s). Middle, CN0-induced activation of dmPFC CRF neurons robustly suppressed task-related activity of strongly tuned delay ( $n=24)$ and reward ( $n=16)$, WS neurons relative to vehicle (delay, $n=25$; reward, $n=15$ ), and CN0-treated viral controls (delay, $n=54$; reward, $n=11$ ). Right, PFC CRF neuronal activation diminished the population size of strongly tuned delay (top), but not reward (bottom) neurons. $\boldsymbol{D}$, Left, Exemplar rasters/PETHs of a WS neuron untuned to delay (top) and reward (bottom) under baseline and CNO conditions. CNO had no significant effects on task-related activity (middle) or population sizes (right) of these neurons in hM3Dq animals (delay, $n=55$; reward, $n=65$ ), relative to vehicle (delay, $n=$ 40; reward, $n=59$ ), and viral controls (delay, $n=64$; reward, $n=92$ ). ${ }^{*} p<0.05$ versus control-CNO. ${ }^{+} p<0.05$ versus hM3Dq-SAL. ${ }^{* * *} p<0.001$ versus control-CNO. ${ }^{+++} p<$ 0.001 versus $\mathrm{hM} 3 \mathrm{Dq}-\mathrm{SAL}$.

and mediodorsal thalamic nucleus (Sesack et al., 1989; Gorelova and Yang, 1997). The neural basis for the topography associated with the cognitive actions of PFC CRF neurotransmission is an important area for future studies.

Neurocircuitry underlying the cognitive actions of PFC CRF neurons

Limited evidence indicates at least some PFC CRF neurons are GABAergic interneurons (Mohila and Onn, 2005; Helmeke et al., 2008). Consistent with this, recent immunohistochemical studies in our laboratory indicate that $\sim 15 \%$ of CRF neurons are immu- noreactive for the GABAergic marker, GAD67, with the remaining $85 \%$ displaying immunoreactivity for the glutamatergic marker, CaMKII $\alpha$. The observation that CRF acts locally in the PFC to impair working memory could implicate GABAergic CRF interneurons in the cognitive effects of our chemogenetic manipulations. However, the fact that PFC CRF neuronal activation had no effects on the broader population of neurons not displaying task-related activity indicates that PFC CRF signaling does not generally increase inhibitory tone within the dmPFC. Ongoing studies in our laboratory are investigating the potential contribution of distinct PFC CRF neuronal subpopulations to working memory. 


\section{A Strongly tuned}

Delay
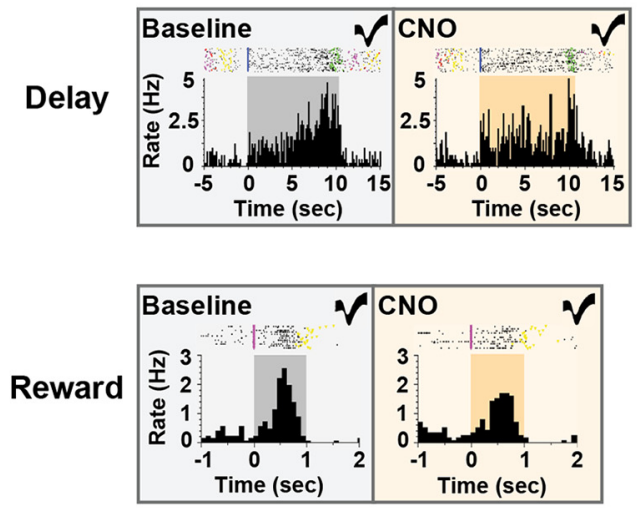

\section{B Untuned}
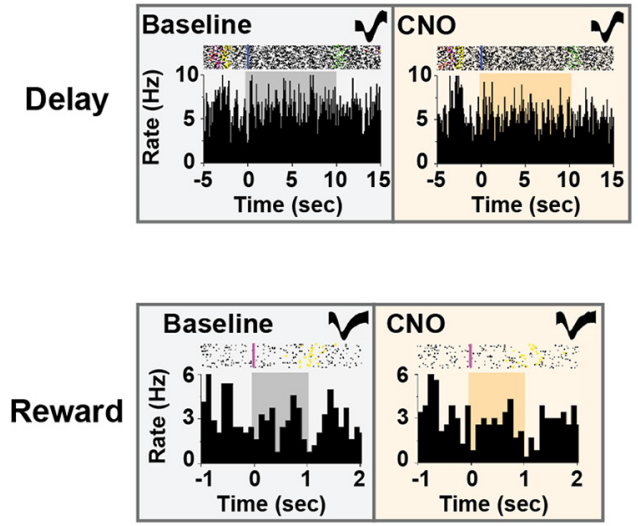
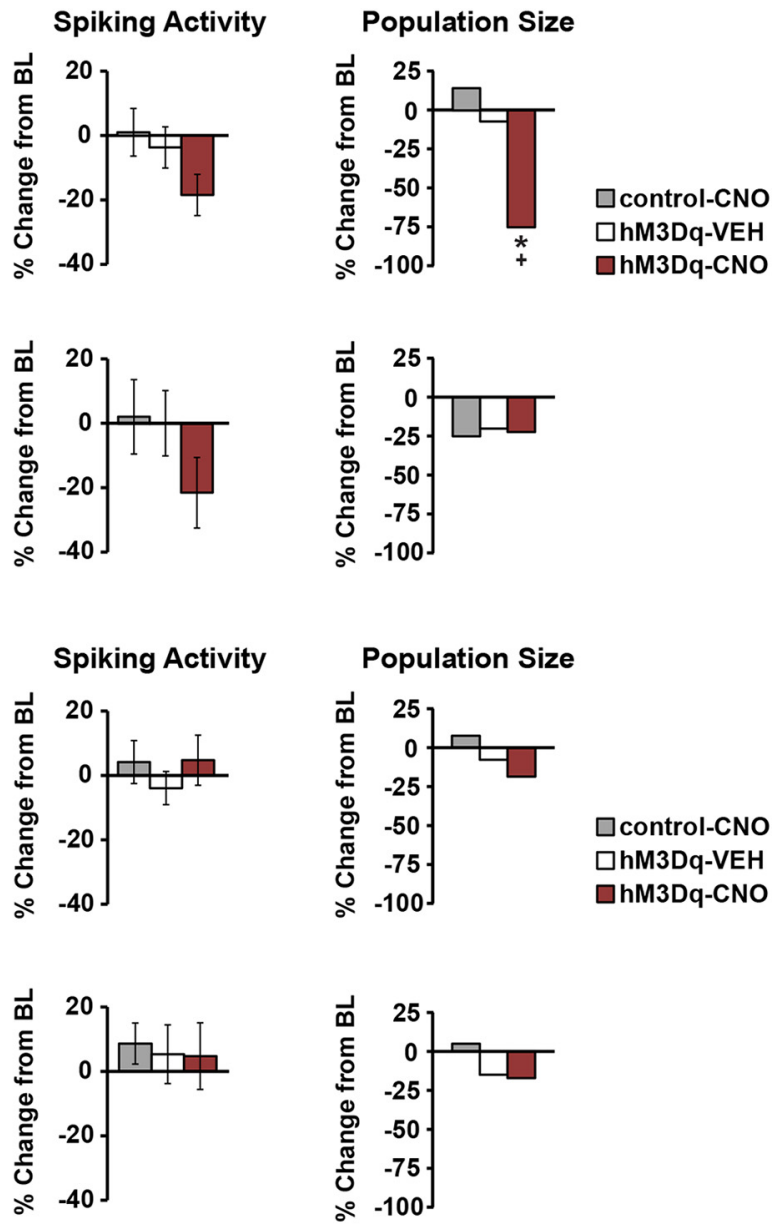

Figure 6. Effects of PFC CRF neuronal activation on task-related activity of dmSTR MSNs. A, Left, Exemplar rasters/PETHs demonstrating task-related activity of strongly tuned delay (top) and reward (bottom) MSNs under baseline and CNO conditions. Middle, CN0 elicited a trend for suppression of task-related activity of strongly tuned delay $(n=12)$ and reward MSNs $(n=9)$ in hM3Dq animals that was not observed with vehicle (delay, $n=28$; reward, $n=15$ ) or CN0-treated viral controls (delay, $n=28$; reward, $n=12$ ). Right, PFC (RF neuronal activation diminished the population size of strongly tuned delay (top), but not reward (bottom), MSNs. B, Left, Exemplar rasters/PETHs of MSNs untuned to delay (top) and reward (bottom) MSNs. CN0 elicited no significant effects on task-related activity (middle) or the population size (right) of untuned MSNs in hM3Dq animals (delay, $n=27$; reward, $n=35$ ), vehicle-treated hM3Dq animals (delay, $n=26$; reward, $n=65$ ), or CN0-treated viral controls (delay, $n=39$; reward, $n=61$ ). ${ }^{*} p<0.05$ versus control-CN0. ${ }^{+} p<0.05$ versus hM3Dq-SAL.

Chemogenetic activation of caudal dmPFC CRF neurons robustly suppressed delay-related activity of dmPFC neurons. This was accompanied by a dramatic decrease in the population size of WS neurons identified as strongly tuned to delay. A weaker, though significant, suppression of reward-related activity was also observed. Consistent with the weaker nature of this effect, we did not observe a significant reduction in the population size of reward-tuned PFC neurons. The neural mechanisms underlying the preferential actions of PFC CRF neurotransmission on delayrelated activity remain to be determined. In the downstream dmSTR, activation of PFC CRF neurons strongly reduced the population of delayed-tuned neurons, but elicited weaker and nonsignificant reductions in the firing rate of delay- and rewardtuned MSNs. Combined, these observations indicate that caudal dmPFC CRF neurons weaken task-related coding broadly, but differentially, within frontostriatal circuitry.

Consistent with prior studies, we observed no mCherry fibers in the dmSTR, suggesting that PFC CRF neurons may not directly innervate the dmSTR (Swanson et al., 1983; Merchenthaler, 1984). Nonetheless, PFC CRF neuronal activation weakened task-related neuronal coding in the dmSTR. It is possible that delay-related activity of dmSTR neurons is driven, at least in part, by excitatory projections from delay and reward-tuned pyrami- dal neurons in the dmPFC. Thus, CRF-dependent reductions in excitatory signaling from the $\mathrm{dmPFC}$ to the $\mathrm{dmSTR}$ may contribute to a weakening of task-related activity in the dmSTR. The weaker effects observed in the dmSTR, relative to the dmPFC, could reflect the fact that task-related activity of MSNs is not solely driven by caudal dmPFC delay-tuned neurons. Indeed, both the rostral and caudal aspects of the dmPFC are known to project to the dmSTR region recorded from in the current studies (Spencer et al., 2012). Thus, neurons in the rostral dmPFC may well contribute to task-related coding in the dmSTR. The precise neural circuitry by which PFC CRF signaling modulates downstream striatal targets remains a topic of future studies.

\section{Translational relevance}

PFC cognitive dysfunction is associated with a variety of behavioral disorders, including ADHD, depression, schizophrenia, and addiction. The fact that CRF acts in the PFC to modulate higher cognitive function could implicate CRF in one or more of these disorders and/or suggest that drugs interfering with CRF neurotransmission may have therapeutic potential for the treatment of PFC-dependent cognitive dysfunction. Additionally, the current observations suggest that the cognition-enhancing actions of caudal dmCRF neuronal suppression mimic those seen with all 
FDA-approved drugs for ADHD (Spencer et al., 2015). Thus, CRF may represent a novel target for the development of pharmacological treatments for this disorder. Beyond these clinical conditions, the cognitive and neurophysiological actions of PFC CRF neuronal activation are similar to those seen with stress and stress-related disorders (Birnbaum et al., 1999; Arnsten, 2009; Devilbiss et al., 2017). Evidence suggests acute stress increases levels of both CRF and CRF1 receptor mRNA in the PFC (Meng et al., 2011; Uribe-Mariño et al., 2016). Additionally, viralmediated deletion of CRF1 receptors prevented acute stressinduced impairment in reversal learning (Uribe-Mariño et al., 2016). Collectively, these observations suggest the hypothesis that stress-related cognitive impairment involves increased CRF neurotransmission in the PFC.

While CRF neurotransmission in the PFC may contribute to stress-related cognitive impairment, in the current studies, animals were highly habituated and motivated to engage in cognitive testing and displayed relatively high baseline performance accuracy. Thus, the cognition-enhancing actions of CRF neuronal suppression are unlikely to reflect solely an "anti-stress" effect. Instead, these results suggest that PFC CRF neurons are active across a range of conditions associated with higher arousal and motivational states, both stressful and nonstressful (Merali et al., 2004). The broader behavioral actions of CRF neurotransmission in the PFC and the translational relevance of these actions are important topics for future research.

\section{References}

Akhlaghpour H, Wiskerke J, Choi JY, Taliaferro JP, Au J, Witten IB (2016) Dissociated sequential activity and stimulus encoding in the dorsomedial striatum during spatial working memory. eLife 5:e19507.

Alsene KM, Rajbhandari AK, Ramaker MJ, Bakshi VP (2011) Discrete forebrain neuronal networks supporting noradrenergic regulation of sensorimotor gating. Neuropsychopharmacology 36:1003-1014.

Arnsten AF (2009) Stress signalling pathways that impair prefrontal cortex structure and function. Nat Rev Neurosci 10:410-422.

Arnsten AF (2011) Catecholamine influences on dorsolateral prefrontal cortical networks. Biol Psychiatry 69:e89-e99.

Batuev AS, Kursina NP, Shutov AP (1990) Unit activity of the medial wall of the frontal cortex during delayed performance in rats. Behav Brain Res 41:95-102.

Berke JD, Okatan M, Skurski J, Eichenbaum HB (2004) Oscillatory entrainment of striatal neurons in freely moving rats. Neuron 43:883-896.

Berridge CW, Arnsten AF (2015) Catecholamine mechanisms in the prefrontal cortex: proven strategies for enhancing higher cognitive function. Curr Opin Behav Sci 4:33-40.

Berridge CW, Schmeichel BE, España RA (2012a) Noradrenergic modulation of wakefulness/arousal. Sleep Med Rev 16:187-197.

Berridge CW, Shumsky JS, Andrzejewski ME, McGaughy JA, Spencer RC, Devilbiss DM, Waterhouse BD (2012b) Differential sensitivity to psychostimulants across prefrontal cognitive tasks: differential involvement of noradrenergic $\alpha 1$ - and $\alpha 2$-receptors. Biol Psychiatry 71:467-473.

Birnbaum S, Gobeske KT, Auerbach J, Taylor JR, Arnsten AF (1999) A role for norepinephrine in stress-induced cognitive deficits: $\alpha$-1-adrenoceptor mediation in the prefrontal cortex. Biol Psychiatry 46:1266-1274.

Chalmers DT, Lovenberg TW, De Souza EB (1995) Localization of novel corticotropin-releasing factor receptor (CRF2) mRNA expression to specific subcortical nuclei in rat brain: comparison with CRF1 receptor mRNA expression. J Neurosci 15:6340-6350.

Charlton BG, Ferrier IN, Perry RH (1987) Distribution of corticotropinreleasing factor-like immunoreactivity in human brain. Neuropeptides 10:329-334.

Compton WM, Volkow ND (2006) Abuse of prescription drugs and the risk of addiction. Drug Alcohol Depend 83 [Suppl 1]:S4-S7.

Cubillo A, Halari R, Ecker C, Giampietro V, Taylor E, Rubia K (2010) Reduced activation and inter-regional functional connectivity of frontostriatal networks in adults with childhood attention-deficit hyperactivity disorder (ADHD) and persisting symptoms during tasks of motor inhibition and cognitive switching. J Psychiatr Res 44:629-639.
Curtis CE, D'Esposito M (2003) Persistent activity in the prefrontal cortex during working memory. Trends Cogn Sci 7:415-423.

Das M, Vihlen CS, Legradi G (2007) Hypothalamic and brainstem sources of pituitary adenylate cyclase-activating polypeptide nerve fibers innervating the hypothalamic paraventricular nucleus in the rat. J Comp Neurol 500:761-776.

De Souza EB, Insel TR, Perrin MH, Rivier J, Vale WW, Kuhar MJ (1985) Corticotropin-releasing factor receptors are widely distributed within the rat central nervous system: an autoradiographic study. J Neurosci 5:3189-3203.

Devilbiss DM, Jenison RL, Berridge CW (2012) Stress-induced impairment of a working memory task: role of spiking rate and spiking history predicted discharge. PLoS Comput Biol 8:e1002681.

Devilbiss DM, Spencer RC, Berridge CW (2017) Stress degrades prefrontal cortex neuronal coding of goal-directed behavior. Cereb Cortex 27:2970-2983.

Fuster J (2015) The prefrontal cortex. San Diego: Academic.

Fuster JM, Alexander GE (1971) Neuron activity related to short-term memory. Science 173:652-654.

Gabbott PL, Warner TA, Jays PR, Salway P, Busby SJ (2005) Prefrontal cortex in the rat: projections to subcortical autonomic, motor, and limbic centers. J Comp Neurol 492:145-177.

Gallopin T, Geoffroy H, Rossier J, Lambolez B (2006) Cortical sources of $\mathrm{CRF}, \mathrm{NKB}$, and CCK and their effects on pyramidal cells in the neocortex. Cereb Cortex 16:1440-1452.

Gorelova N, Yang CR (1997) The course of neural projection from the prefrontal cortex to the nucleus accumbens in the rat. Neuroscience 76:689706.

Heidbreder CA, Groenewegen HJ (2003) The medial prefrontal cortex in the rat: evidence for a dorso-ventral distinction based upon functional and anatomical characteristics. Neurosci Biobehav Rev 27:555-579.

Helmeke C, Ovtscharoff W Jr, Poeggel G, Braun K (2008) Imbalance of immunohistochemically characterized interneuron populations in the adolescent and adult rodent medial prefrontal cortex after repeated exposure to neonatal separation stress. Neuroscience 152:18-28.

Histed MH, Pasupathy A, Miller EK (2009) Learning substrates in the primate prefrontal cortex and striatum: sustained activity related to successful actions. Neuron 63:244-253.

Horst NK, Laubach M (2013) Reward-related activity in the medial prefrontal cortex is driven by consumption. Front Neurosci 7:56.

Hupalo S, Berridge CW (2016) Working memory impairing actions of corticotropin-releasing factor (CRF) neurotransmission in the prefrontal cortex. Neuropsychopharmacology 41:2733-2740.

Hyman JM, Zilli EA, Paley AM, Hasselmo ME (2010) Working memory performance correlates with prefrontal-hippocampal theta interactions but not with prefrontal neuron firing rates. Front Integr Neurosci 4:2.

Kim YB, Huh N, Lee H, Baeg EH, Lee D, Jung MW (2007) Encoding of action history in the rat ventral striatum. J Neurophysiol 98:3548-3556.

Levy R, Friedman HR, Davachi L, Goldman-Rakic PS (1997) Differential activation of the caudate nucleus in primates performing spatial and nonspatial working memory tasks. J Neurosci 17:3870-3882.

Lewis DA, Foote SL, Cha CI (1989) Corticotropin-releasing factor immunoreactivity in monkey neocortex: an immunohistochemical analysis. J Comp Neurol 290:599-613.

Liston C, Malter Cohen M, Teslovich T, Levenson D, Casey BJ (2011) Atypical prefrontal connectivity in attention-deficit/hyperactivity disorder: pathway to disease or pathological end point? Biol Psychiatry 69:11681177.

Mailly P, Aliane V, Groenewegen HJ, Haber SN, Deniau JM (2013) The rat prefrontostriatal system analyzed in 3D: evidence for multiple interacting functional units. J Neurosci 33:5718-5727.

Meng QY, Chen XN, Tong DL, Zhou JN (2011) Stress and glucocorticoids regulated corticotropin releasing factor in rat prefrontal cortex. Mol Cell Endocrinol 342:54-63.

Merali Z, McIntosh J, Anisman H (2004) Anticipatory cues differentially provoke in vivo peptidergic and monoaminergic release at the medial prefrontal cortex. Neuropsychopharmacology 29:1409-1418.

Merchenthaler I (1984) Corticotropin releasing factor (CRF)-like immunoreactivity in the rat central nervous system. extrahypothalamic distribution. Peptides 5 [Suppl 1]:53-69.

Miguel TT, Gomes KS, Nunes-de-Souza RL (2014) Tonic modulation of anxiety-like behavior by corticotropin-releasing factor (CRF) type 1 re- 
ceptor (CRF1) within the medial prefrontal cortex (mPFC) in male mice: role of protein kinase A (PKA). Horm Behav 66:247-256.

Mitchell JF, Sundberg KA, Reynolds JH (2007) Differential attentiondependent response modulation across cell classes in macaque visual area V4. Neuron 55:131-141.

Mohila CA, Onn SP (2005) Increases in the density of parvalbuminimmunoreactive neurons in anterior cingulate cortex of amphetaminewithdrawn rats: evidence for corticotropin-releasing factor in sustained elevation. Cereb Cortex 15:262-274.

Olschowka JA, O’Donohue TL, Mueller GP, Jacobowitz DM (1982) The distribution of corticotropin releasing factor-like immunoreactive neurons in rat brain. Peptides 3:995-1015.

Povysheva NV, Gonzalez-Burgos G, Zaitsev AV, Kröner S, Barrionuevo G, Lewis DA, Krimer LS (2006) Properties of excitatory synaptic responses in fast-spiking interneurons and pyramidal cells from monkey and rat prefrontal cortex. Cereb Cortex 16:541-552.

Rajbhandari AK, Baldo BA, Bakshi VP (2015) Predator stress-induced CRF release causes enduring sensitization of basolateral amygdala norepinephrine systems that promote PTSD-like startle abnormalities. J Neurosci 35:14270-14285.

Rigotti M, Barak O, Warden MR, Wang XJ, Daw ND, Miller EK, Fusi S (2013) The importance of mixed selectivity in complex cognitive tasks. Nature 497:585-590.

Scharner S, Prinz P, Goebel-Stengel M, Kobelt P, Hofmann T, Rose M, Stengel A (2016) Activity-based anorexia reduces body weight without inducing a separate food intake microstructure or activity phenotype in female rats: mediation via an activation of distinct brain nuclei. Front Neurosci 10:475.

Schultz W, Romo R (1988) Neuronal activity in the monkey striatum during the initiation of movements. Exp Brain Res 71:431-436.

Senut MC, Menetrey D, Lamour Y (1989) Cholinergic and peptidergic projections from the medial septum and the nucleus of the diagonal band of
Broca to dorsal hippocampus, cingulate cortex and olfactory bulb: a combined wheatgerm agglutinin-apohorseradish peroxidase-gold immunohistochemical study. Neuroscience 30:385-403.

Sesack SR, Deutch AY, Roth RH, Bunney BS (1989) Topographical organization of the efferent projections of the medial prefrontal cortex in the rat: an anterograde tract-tracing study with phaseolus vulgaris leucoagglutinin. J Comp Neurol 290:213-242.

Setlik J, Bond GR, Ho M (2009) Adolescent prescription ADHD medication abuse is rising along with prescriptions for these medications. Pediatrics 124:875-880.

Spencer RC, Klein RM, Berridge CW (2012) Psychostimulants act within the prefrontal cortex to improve cognitive function. Biol Psychiatry 72:221-227.

Spencer RC, Devilbiss DM, Berridge CW (2015) The cognition-enhancing effects of psychostimulants involve direct action in the prefrontal cortex. Biol Psychiatry 77:940-950.

Stalnaker TA, Calhoon GG, Ogawa M, Roesch MR, Schoenbaum G (2012) Reward prediction error signaling in posterior dorsomedial striatum is action specific. J Neurosci 32:10296-10305.

Swanson LW, Sawchenko PE, Rivier J, Vale WW (1983) Organization of ovine corticotropin-releasing factor immunoreactive cells and fibers in the rat brain: an immunohistochemical study. Neuroendocrinology 36:165-186.

Uribe-Mariño A, Gassen NC, Wiesbeck MF, Balsevich G, Santarelli S, Solfrank B, Dournes C, Fries GR, Masana M, Labermeier C, Wang XD, Hafner K, Schmid B, Rein T, Chen A, Deussing JM, Schmidt MV (2016) Prefrontal cortex corticotropin-releasing factor receptor 1 conveys acute stress-induced executive dysfunction. Biol Psychiatry 80:743-753.

Voorn P, Vanderschuren LJ, Groenewegen HJ, Robbins TW, Pennartz CM (2004) Putting a spin on the dorsal-ventral divide of the striatum. Trends Neurosci 27:468-474. 